\title{
MODULES OVER SOME GROUP RINGS, HAVING $d$-GENERATOR PROPERTY
}

\author{
V.A. BOVDI AND L.A. KURDACHENKO
}

\begin{abstract}
For modules over group rings we introduce the following numerical parameter. We say that a module $A$ over a ring $R$ has finite $r$-generator property if each f.g. (finitely generated) $R$-submodule of $A$ can be generated exactly by $r$ elements and there exists a f.g. $R$-submodule $D$ of $A$, which has a minimal generating subset, consisting exactly of $r$ elements. Let $F G$ be the group algebra of a finite group $G$ over a field $F$. In the present paper modules over the algebra $F G$ having finite generator property are described.
\end{abstract}

\section{INTRODUCTION AND MAIN RESULTS}

Let $R$ be an associate ring and let $A$ be a module over the ring $R$. The structure and the properties of $A$ essentially depend on the properties of the family of its finitely generated submodules. Clearly, if $B$ is a finitely generated submodule of $A$ then $B$ can have different generator systems. Therefore, it makes sense to consider a minimal system of generators. Recall that a subset $M \subseteq B$ is called a minimal generating subset for $B$ if $M$ generates $B$ and each proper subset of $M$ generates a proper submodule of $B$. If $R$ is a field and $A$ is a vector space, then the number of elements in each minimal system of generators for $B$ is the same, i.e. it is an invariant of $B$. Generally such a situation does not always take place. Moreover, there are not so many rings for which this holds. Therefore, in the submodule $B$, we consider those minimal systems of generators that have the least number of elements. The number of elements in such minimal system of generators is denoted by $d_{R}(A)$.

If $A$ is a vector space over a field $F$, then $d_{F}(A)$ is exactly the dimension of $A$. However, when moving from vector spaces to modules, the properties of the numerical invariant $d_{R}(A)$ can already differ significantly from the classical properties of the dimensions of vector subspaces. For example, if $B$ is a proper subspace of the the vector space $A$, then $\operatorname{dim}_{F}(B)<\operatorname{dim}_{F}(A)$. Unlike vector spaces, not each submodule of a finitely generated module is finitely generated. Moreover, in the case when a submodule is finitely generated it can has a minimal generating set with more

2020 Mathematics Subject Classification. 20C05; 20F50 Secondary: 20D25; 16D10; 16D70; $16 \mathrm{D} 80$

Key words and phrases. module over a group ring, $r$-generator property, width of a module.

The research was supported by the UAEU UPAR Grant G00002160. 
elements than the number of elements in the generating system of the entire module. The following simple example illustrates this situation.

Let $p$ be a prime. Let $G=\langle g\rangle \times\langle h\rangle \cong C_{p} \times C_{p}$ be a multiplicative $p$-group of order $p^{2}, A=\langle b\rangle \oplus\langle c\rangle \oplus\langle d\rangle \cong \mathbb{Z}_{p} \oplus \mathbb{Z}_{p} \oplus \mathbb{Z}_{p}$ an additive elementary abelian $p$-group of order $p^{3}$ and let $H=\langle c\rangle \oplus\langle d\rangle \leq A$. Define a right action $\circ$ of $g$ and $h$ on $A$ (concerning the basis $\{b, c, d\}$ ) by the following rule:

$$
a \circ g=\left(\begin{array}{l}
b \\
c \\
d
\end{array}\right)\left(\begin{array}{lll}
1 & 0 & 0 \\
1 & 1 & 0 \\
0 & 0 & 1
\end{array}\right) \quad \text { and } a \circ h=\left(\begin{array}{l}
b \\
c \\
d
\end{array}\right)\left(\begin{array}{lll}
1 & 0 & 0 \\
0 & 1 & 0 \\
1 & 0 & 1
\end{array}\right), \quad(a \in A) \text {. }
$$

In other words $b \circ g=b+c, c \circ g=c, d \circ g=d, b \circ h=b+d, c \circ h=c$ and $d \circ h=d$. Clearly, $A=\langle b\rangle_{\mathbb{Z}_{p} G}$ is a cyclic right $\mathbb{Z}_{p} G$-module, in which $d_{\mathbb{Z}_{p} G}(A)=1$, but $d_{\mathbb{Z}_{p} G}(H)=2$.

Each ring $R$ and their ideals are itself the modules over $R$. Therefore, it is natural to consider first the number of generating elements (left or right) ideals in the ring $R$. It is clear that it makes sense to do this for the rings whose (left or right) ideals are finitely generated, i.e. for the Noetherian rings. In [7] those commutative rings $R$ were considered, for which there exists a positive integer $r$ such that $d_{R}(I) \leq r$ for each ideal $I$ of $R$. It is immediately clear that all examples of such rings are principal ideal domains and Dedekind domains. Commutative rings with such property actively studied in [7, 14, 15, 16, 18, 21, 22, 23]. Gilmer [14] generalized this situation, where he considered commutative rings for which there exists a positive integer $r$ such that $d_{R}(I) \leq r$ for every finitely generated ideal $I$ of $R$. Such rings are called rings with $r$ - generator property. The most famous examples of such rings (see, for example, [13, Chapter II.1, Chapter III.5]) are valuation domains and Bezout domains (i.e. domains in which every finitely generated ideal is principal). Commutative rings having $r$-generator property have been studied in [14, 15, 16, 18, 21, 22, 23]. In group theory, an analogue of this concept arose earlier in the work of Maltsev [20]. Following Maltsev, we say that a group $G$ has finite special rank $r$, where $r$ is a positive integer, if $d(H) \leq r$ for every finitely generated subgroup $H$ of the group $G$. In group theory this concept turned out to be very useful and productive (for example, see the survey [10] and the book [11]). Thus, it is a native to consider an analog of this natural concept for modules as well. Let $A$ be a module over a ring $R$. We say that $A$ has $d$-R-generator property if there exists a positive integer $d$ such that $d_{R}(B) \leq d$ for each finitely generated $R$-submodule $B$ of $A$. If we consider only one fixed ring $R$, then we simply say that $A$ has $d$-generator property.

In [5] modules were studied which have $d$-generator property over the group algebra $F G$, where $F$ is a field of characteristic 0 and $G$ is a finite group. In the paper mentioned above we used another term - "special rank", but in ring theory there are already several terms using the word rank, so in this paper we preferred to use the term introduced by R. Gilmer for the rings. 
In the present paper we study modules having $d$-generator property over the group algebras $F G$ where $F$ is a field of arbitrary characteristic and $G$ is a finite group. The main results are the following.

Theorem 1. Let $r \in \mathbb{N}$ and let $F G$ be the group algebra of a finite group $G$ over a field F. An FG-module A has a finite r-generator property if and only if the following conditions hold:

(i) $\operatorname{dim}_{F}(A)$ is finite;

(ii) each semisimple homogeneous FG-factor of $A$ has width at most $r$ and there exists a semisimple homogeneous FG-factor, whose width is exactly $r$.

Theorem 2. Let $n, r \in \mathbb{N}$ and let $F G$ be the semisimple group algebra of a finite group $G$ over a field $F$ of prime characteristic $p$. An FG-module $A$ has finite $r$-generator property if and only if the following conditions hold:

(i) $A=\oplus_{j=1}^{n} H_{j}$ in which each $H_{j}=\oplus_{k=1}^{t(j)} D_{k}$, where each $D_{k}$ is a simple $F G$ submodule of $A,|G| \leq t(j)$ and $D_{k} \cong_{F G} D_{m}$ for all $1 \leq k, m \leq t(j)$;

(ii) $t(j) \leq r$ for all $j$ and there exists $s \in \mathbb{N}$ such that $t(s)=r$;

(iii) $n \leq n n s_{F}(G)$, where $n n s_{F}(G)$ is the number of pairwise non-isomorphic simple FG-modules.

Theorem 3. Let $m, r \in \mathbb{N}$ and let $F G$ be the modular group algebra of a finite group $G$ over a field $F$ of prime characteristic $p$. Let $P \in S_{y} l_{p}(G)$ be a normal Sylow $p$ subgroup of $G$. If an FG-module A has finite $r$-generator property, then the following conditions hold:

(i) $\operatorname{dim}_{F}(A)$ is finite;

(ii) A has a series of FG-submodules

$$
\langle 0\rangle=Z_{0} \leq Z_{1} \leq \cdots \leq Z_{m-1} \leq Z_{m}=A
$$

such that $m \leq|P|$ and each $G / C_{G}\left(Z_{j+1} / Z_{j}\right)$ is a p-group, each factor $Z_{j+1} / Z_{j}$ is a semisimple FG-module, whose homogeneous components have width at most $r$ and the number of homogeneous components is at most nns $s_{F}(G / P)$, where $\mathrm{nns}_{F}(G)$ is the number of pairwise non-isomorphic simple FG-modules.

\section{Preliminaries AND Lemmas}

In the sequel, we use the notions and results from the books [4, 9, 11].

Lemma 2.1. Let $A$ be an $R$-module over a ring $R$ such that $A$ has finite $r$-generator property. If $B, C$ are $R$-submodules of $A$ such that $B \leq_{R} C$ then the following conditions hold:

(i) the submodule $B$ has finite r-generator property at most $r$;

(ii) the factor-module $A / B$ has finite $r$-generator property at most $r$;

(iii) the factor-module $C / B$ has finite $r$-generator property at most $r$. 
These assertions are obvious.

Lemma 2.2. Let $r, t \in \mathbb{N}$ and let $B$ be a submodule of an $R$-module $A$ over a Noetherian ring $R$. If $B$ has finite $r$-generator property at most $r$ and $A / B$ has finite $d$-generator property at most $t$, then $A$ has $d$-generator property at most $r+t$.

Proof. Let $D$ be a f.g. $R$-submodule of $A$. Clearly, $D$ is Noetherian as an f.g. module (see [19, Lemma 1.1]). The factor $D /(D \cap B) \cong(D+B) / B$ has finite generating subset $\left\{d_{j}+(D \cap B) \mid 1 \leq j \leq m\right\}$ in which $m \leq t$. It follows that $D \cap B$ is a f.g. $R$-submodule of $B$. Since $B$ has $r$-generator property, $D \cap B$ has finite generating subset $\left\{b_{j} \mid 1 \leq j \leq k\right\}$ where $k \leq r$. The subset $\left\{d_{j} \mid 1 \leq j \leq m\right\} \cup\left\{b_{j} \mid 1 \leq j \leq k\right\}$ generates $D$ as an $R$-submodule and $m+k \leq t+r$.

Lemma 2.3. Let $A=\oplus_{j=1}^{n} A_{j}$ be an $R$-module over a ring $R$ in which each $A_{j}$ is a simple $R$-submodule. If elements of the set $\left\{A n n_{R}^{\text {left }}\left(A_{1}\right), \ldots\right.$, Ann $\left.n_{R}^{\text {left }}\left(A_{n}\right)\right\}$ are pairwise non-isomorphic, then $A$ is a cyclic $R$-module.

Proof. Let us prove that $A=\left\langle a_{1}+\cdots+a_{n} \mid 0 \neq a_{j} \in A_{j}\right\rangle_{R}$. Since each $A_{j}$ is a simple $R$-module, $U_{j}:=A n n_{R}^{\text {left }}\left(A_{j}\right)$ is a maximal left ideal of $R$. The fact that a sum of left ideals is also a left ideal together with $U_{j} \neq U_{m}$ implies that $U_{j}+U_{m}=R$ for all $j \neq m$. Thus $U_{1}+U_{2}=R$ and there exists $\alpha \in U_{2} \backslash U_{1}$ such that $\alpha a_{1} \neq 0, \alpha a_{2}=0$ and

$$
\alpha\left(a_{1}+\cdots+a_{n}\right)=\alpha a_{1}+\left(\alpha a_{3}+\cdots+\alpha a_{n}\right) .
$$

If $\alpha a_{3}+\cdots+\alpha a_{n}=0$, then $\alpha a_{1} \in R\left(a_{1}+\cdots+a_{n}\right)$. Since $A_{1}$ is a simple $R$-module, it can be generated by each of its non-zero elements, so $A_{1}=\left\langle a_{1}\right\rangle_{R} \leq\left\langle a_{1}+\cdots+a_{n}\right\rangle_{R}$.

Let $\alpha a_{3}+\cdots+\alpha a_{n} \neq 0$ and let $j_{1} \in \mathbb{N}$ be a smallest integer (say $j_{1}=3$ ) such that $j_{1}>2$ and $\alpha A_{j_{1}} \neq 0$. The equality $U_{1}+U_{3}=R$ shows that there exists $\beta \in U_{3} \backslash U_{1}$ such that $\beta \alpha a_{1} \neq 0, \beta \alpha a_{3}=0$ and

$$
\beta\left(\alpha a_{1}+\alpha a_{3}+\cdots+\alpha a n\right)=\beta \alpha a_{1}+\left(\beta \alpha a_{4}+\cdots+\beta \alpha a_{n}\right) .
$$

If $\beta \alpha a_{4}+\cdots+\beta \alpha a_{n}=0$, then, as above, $A_{1} \leq\left\langle a_{1}+\cdots+a_{n}\right\rangle_{R}$. Otherwise, all this process we can repeat again. Since $n \in \mathbb{N}$, after finitely many steps we obtain that $A_{1} \leq R\left(a_{1}+\cdots+a_{n}\right)$ and $A=A_{1} \oplus \cdots \oplus A_{n}=\left\langle a_{1}+\cdots+a_{n}\right\rangle_{R}$.

Lemma 2.4. Let $A=\oplus_{j=1}^{n} A_{j}$ be an $R$-module over a ring $R$ in which each $A_{j}$ is a simple $R$-submodule. If all elements of the set $\left\{\operatorname{Ann}_{R}^{\text {left }}\left(A_{1}\right), \ldots, \operatorname{Ann}_{R}^{\text {left }}\left(A_{n}\right)\right\}$ coincide, then $A n n_{R}^{\text {left }}\left(A_{j}\right)=A n n_{R}^{\text {left }}\left(\langle a\rangle_{R}\right)$ for each $a \in A \backslash\{0\}$. In particular, the $R$-submodule $\langle a\rangle$ is isomorphic to each $A_{j}$ for $1 \leq j \leq n$.

Proof. Set $L:=A n n_{R}^{\text {left }}\left(A_{1}\right)$. Clearly, $L \leq A n n_{R}^{\text {left }}(a)$ for each $0 \neq a \in A$, so that $L \leq A n n_{R}^{\text {left }}(A)$. Since $A_{1}$ is a simple $R$-module, $L$ is a maximal left ideal of $R$. It follows that $L=A n n_{R}^{\text {left }}(A)$.

Let $y \in R \backslash L$ and let $a=a_{1}+\cdots+a_{n} \in A \backslash\{0\}$, in which each $a_{j} \in A_{j}, a_{1} \neq 0$ and $\operatorname{Ann}_{R}^{\text {left }}(a)=R$. Since $y a=0$ and $a_{1}, \ldots, a_{n}$ are linearly independent over $R$, 
$y A_{j}=0$ for each $j$. In particular, $y a_{1}=0$ and $y \in A n n_{R}^{\text {left }}\left(A_{1}\right)=L$, a contradiction. It follows that $A n n_{R}^{\text {left }}(a) \neq R$ for each $a \in R \backslash\{0\}$. Consequently, $\operatorname{Ann}_{R}^{\text {left }}(a)=L$ and $\langle b\rangle_{R} \cong_{R} R / L$ for each $b \in R \backslash\{0\}$.

Corollary 2.5. Let $\Lambda$ be a set and let $A=\oplus_{\lambda \in \Lambda} A_{\lambda}$ be an $R$-module over a ring $R$ in which each $A_{\lambda}$ is a simple $R$-submodule. If

$$
A n n_{R}^{l e f t}\left(A_{\lambda}\right)=A n n_{R}^{\text {left }}(A \mu), \quad(\lambda, \mu \in \Lambda)
$$

then each cyclic $R$-submodule of $A$ is simple and $A_{\lambda} \cong_{R}\langle a\rangle_{R}$ for each $a \in A \backslash\{0\}$.

Corollary 2.6. Let $n, r \in \mathbb{N}$ and let $A=\oplus_{j=1}^{n} A_{j}$ be an $R$-module over a ring $R$ in which each $A_{j}$ is a simple $R$-submodule.

If all elements of the set $\left\{A n n_{R}^{\text {left }}\left(A_{1}\right), \ldots, A n n_{R}^{\text {left }}\left(A_{n}\right)\right\}$ coincide, then the following conditions are equivalent:

(i) $d_{R}(A)=r$;

(ii) A has finite r-generator property;

(iii) $n=r$, where $n=\operatorname{width}_{R}(A)$.

Proof. $(i) \Leftrightarrow($ iii $)$. Let $S:=\left\{b_{1}, \ldots, b_{k}\right\} \subseteq A$ be a minimal set, such that $A=\langle S\rangle_{R}$ and let $B_{j}:=\left\langle b_{j}\right\rangle_{R}$. Obviously, $B_{j} \cong_{R} A_{j}$ after a suitable renumbering of the set $\left\{A_{k}\right\}$ and $B_{j}$ is simple for each $j$ by Lemma 2.4. Since $A$ is a semisimple $R$-module, $A$ includes an $R$-submodule $D_{1}$ such that $A=B_{1} \oplus D_{1}$ (see [19, Corollary 4.3]). If $b_{t} \in B_{1}$ for some $t>1$, then $A=\left\langle S \backslash b_{t}\right\rangle_{R}$, a contradiction. This yields that $b_{t} \notin B_{1}$. Similarly, if $t>2$ then $b_{t} \notin B_{1} \oplus B_{2}$. Repeating this process finitely many times, we obtain $b_{k} \notin B_{1} \oplus \cdots \oplus B_{k-1}$ and $A=\oplus_{j=1}^{k} B_{j}$.

If $T:=\left\{d_{1}, \ldots, d_{m}\right\} \subseteq A$, such that $S \neq T$ and $A=\langle T\rangle_{R}$, then repeating the above arguments, we obtain a decomposition $A=\oplus_{j=1}^{m} D_{j}$ in which each $D_{j}=\left\langle d_{j}\right\rangle_{R} \cong_{R} A_{j}$ after a suitable renumbering of the set $\left\{A_{k}\right\}$. It follows that $k=m$ by the KrullRemak-Schmidt theorem (see [8, Chapter 6, Theorem 1.6]) and $d_{R}(A)=n$.

$($ ii $) \Leftrightarrow($ iii $)$. Since $A$ is a f.g. $R$-module, $d_{R}(A)=r$. Thus $n=r$ by the part above.

Conversely, let $n=r$. Using part (i) we obtain that $d_{R}(A)=r$. Let B be a f.g. $R$-submodule of A. Since $A$ is a semisimple $R$-module, there exists an $R$-submodule $\mathrm{C}$ such that $A=B \oplus C$ (see [19, Corollary 4.3]). Since $B, C$ are semisimple, $B=\oplus_{j=1}^{s} B_{j}$ and $C=\oplus_{j=1}^{t} C_{j}$, where each $B_{j}$ and $C_{j}$ are simple $R$-submodules (see [19, Corollary 4.3]). Consequently, using the Krull-Remak-Schmidt theorem, we get

$$
A=\left(\oplus_{j=1}^{s} B_{j}\right) \oplus\left(\oplus_{j=1}^{t} C_{j}\right),
$$

in which $s+t=r$ and $B_{j} \cong_{R} A_{1}$, so $d_{R}(B)=s \leq r$ by part (i).

Let $A=\oplus_{j=1}^{n} A_{j}$ be an $R$-module over a ring $R$ in which each $A_{j}$ is a simple $R$-module. The relation " $A_{j} \cong_{R} A_{m}$ " is an equivalence relation on the set $\left\{A_{j} \mid\right.$ $1 \leq j \leq n\}$. Let $E_{1}, \ldots, E_{k}$ be all equivalence classes by its relation. A direct sum 
of all submodules from the set $E_{j}$ we denote by $S_{j}$ and it is called a homogeneous component of $A$.

Corollary 2.7. Let $n, r \in \mathbb{N}$ and let $A=\oplus_{j=1}^{n} A_{j}$ be a semisimple $R$-module over a ring $R$ in which each $A_{j}$ is a simple $R$-module. The following conditions are equivalent:

(i) $d_{R}(A)=r$;

(ii) A has r-generator property;

(iii) all homogeneous components of $A$ have width at most $r$ and there exists a homogeneous component, having width exactly $r$.

Proof. $(i) \Leftrightarrow($ iii $)$. Let $d_{R}(A)=r$. Let $\left\{E_{1}, \ldots, E_{k}\right\}$ be a collection of all homogeneous components of $A$. Set $D_{m}=\oplus_{j=1, j \neq m}^{n} E_{j}$. Clearly, $A=\bigoplus_{j=1}^{k} E_{j}$ and $A / D_{m} \cong_{K} E_{m}$ is a semisimple homogeneous module, so

$$
d_{R}\left(A / D_{m}\right) \leq d_{R}(A)=r, \quad(1 \leq m \leq n)
$$

by Lemma 2.1(ii). Since $d_{R}\left(E_{m}\right)=$ width $_{R}\left(E_{m}\right)$ by Corollary 2.6(i)(iii),

$$
\operatorname{width}_{R}\left(E_{m}\right) \leq r .
$$

Without lost of generality we can assume that $E_{1}$ has a greatest width, say width $_{R}\left(E_{1}\right)=s$ with $s \in \mathbb{N}$. Furthermore, $E_{1}=\oplus_{j=1}^{s} C_{j}$ in which each $C_{j}$ is a simple $R$-submodule whose homogeneous components have width 1 by Corollary 2.6(iii). Each module $C_{j}$ is cyclic by Lemma 2.4, so $A$ has a set of generators consisting of $s$ elements. Hence $s \leq r$. The above proved inequality $r \leq s$ implies that $r=s$. Using similar arguments, the conditions of our lemma are sufficient.

$($ ii $) \Leftrightarrow($ iii $)$. Since $A$ is a f.g. $R$-module, $d_{R}(A)=r$, so we can repeat the proof of part $(i) \Rightarrow($ iii $)$.

Conversely, $($ iii $) \Rightarrow(i)$ as was proved before, so $d_{R}(A)=r$. Let $B \leq_{R} A$ be a f.g. $R$-submodule. Since $A$ is a semisimple $R$-module, there exists an $R$-submodule $C$ such that $A=B \oplus C$ (see [19, Corollary 4.3]) with semisimple components $B$ and $C$ (see [19, Corollary 4.3]). Thus

$$
A=B \oplus C=\left(\oplus_{j=1}^{s} B_{j}\right) \oplus\left(\oplus_{j=1}^{t} C_{j}\right),
$$

where each $B_{j}$ and $C_{j}$ are simple $R$-submodules. Finally, according to the KrullRemak-Schmidt theorem (see [8, Chapter 6, Theorem 1.6]), each homogeneous component of $B$ has width at most $r$, so $d_{R}(B) \leq r$ by part (iii).

Lemma 2.8. Let $A$ be an $F G$-module, where $F G$ is the group algebra of a finite group $G$ over a field $F$. If $A$ has finite $r$-generator property, then $\operatorname{dim}_{F}(A) \leq r|G|$.

Proof. Let $0 \neq a \in A$. Clearly, $\langle a\rangle_{F G} \cong_{F} F G / A n n_{F G}^{\text {left }}(a)$ and

$$
\operatorname{dim}_{F G}\left(\langle a\rangle_{F G}\right)=\operatorname{dim}_{F}((F G) a) \leq \operatorname{dim}_{F}(F G)=|G| .
$$


Let $B_{1} \leq_{F G} A$ such that $B_{1}$ is f.g. and $B_{1} \neq A$. Since $A$ has $r$-generator property, there exists a finite subset $M=\left\{a_{1}, \ldots, a_{m}\right\}$ such that $B_{1}=\langle M\rangle_{F G}$ and $m \leq r$. Thus

$$
\operatorname{dim}_{F}(B) \leq \operatorname{dim}_{F}\left((F G) a_{1}\right)+\cdots+\operatorname{dim}_{F}\left((F G) a_{m}\right) \leq m|G| \leq r|G|
$$

If $0 \neq d \in A \backslash B$, then $B_{2}:=B_{1}+(F G) d$ is a f.g. module, such that $B_{1} \leq B_{2}$ but $B_{1} \neq B_{2}$. Similarly, continuing this process, we construct a family of proper submodules $\left\{B_{k}<A \mid k \in \mathbb{N}\right\}$ such that each $\operatorname{dim}_{F}\left(B_{k}\right) \leq r|G|$. Consequently, there exists $m \in \mathbb{N}$ such that $B_{m}=A$, so $A$ is a f.g. module and $\operatorname{dim}_{F}(A) \leq r|G|$.

The intersection of all maximal $R$-submodules of an $R$-module $A$ over a $\operatorname{ring} R$ is called the Frattini submodule of $A$ and denoted by $\operatorname{Fratt}_{R}(A)$. For finitely generated modules, the intersection of all of maximal submodules is also called the radical of the module by analogy with the Jacobson radical for rings. Recently, however, some other radicals have appeared in modules, therefore we followed the example of Hartley [17] and called the intersection of all maximal submodules the Frattini submodule.

We freely use the fact that $\operatorname{Fratt}_{R}(A)$ consists of non-generating elements of $A$.

Lemma 2.9. Let $A=\langle M\rangle$ be an $R$-module over a ring $R$. If $S$ is a finite subset of $M$ such that $S \subseteq \operatorname{Fratt}_{R}(A)$, then $A=\langle M \backslash S\rangle$.

Proof. First, assume that $S=\{d\}$. Suppose the contrary, let the subset $M \backslash\{d\}$ generate a proper $R$-submodule $B$. Clearly $d \notin B$. Let $\mathfrak{S}(B)$ be a family of all $R$-submodules of $A$ such that each member of $\mathfrak{S}(B)$ includes $B$ and does not contain the element $d$. Clearly, $B \subseteq \mathfrak{S}(B) \neq \emptyset$ and $\mathfrak{S}(B)$ has a maximal element $C$ by Zorn's lemma. If $D$ is an $R$-submodule of $A$ such that $C \leq D$ and $C \neq D$, then $d \in D$. However, in this case $M \leq D$, which implies that $D=A$. Consequently, $C$ is a maximal $R$-submodule of $\bar{A}$. On the other hand, $d \notin C$, so we get a contradiction with a choice of $d$, which proves the result. The rest follows by induction on $|S|$.

Lemma 2.10. Let $A$ be a Noetherian $R$-module over a ring $R$. If $M \subseteq A$ such that $A / \operatorname{Fratt}_{R}(A)=\left\langle a+\operatorname{Fratt}_{R}(A) \mid a \in M\right\rangle_{R}$, then $A=\langle M\rangle_{R}$ and

$$
d_{R}(A)=d_{R}\left(A / \operatorname{Fratt}_{R}(A)\right) .
$$

Note that, outwardly, our Lemma 2.10 is very similar to the following consequence of the Nakayama's lemma [12, Corollary 4.8(b), p. 124]. In the Nakayama's lemma [12, Corollary 4.8(b), p. 124], was considered a submodule, obtained by acting on the entire module by the radical of the ring. However in our Lemma 2.10 we consider a factor-module by the radical of the module, i.e. at the intersection of all maximal submodules. We provide the following simple example:

Let $A$ be a cyclic group of order $p>2$ which is considered as a module over the ring of integers $\mathbb{Z}$. The radical (or the Frattini submodule) $\operatorname{Fratt}_{\mathbb{Z}}(A)$ of the module $A$ is a subgroup of order $p$ and the radical $\mathcal{J}(\mathbb{Z})$ of the ring of integers $\mathbb{Z}$ is zero. Consequently, $\mathcal{J}(\mathbb{Z}) A=0$. 
Proof of Lemma 2.10. Let $B=\langle M\rangle_{R} \leq_{R} A$ such that $A \neq B$. The choice of $M$ shows that $A=B+D$. Let $B_{1} \leq_{R} A$ be generated by $M \cup\left\{a_{1}\right\}$, where $a_{1} \in D \backslash B$ and $B_{1} \neq A$. Set $B_{2}=\left\langle M \cup\left\{a_{1}\right\} \cup\left\{a_{2}\right\}\right\rangle_{R}$ such that $a_{2} \in D \backslash B_{1}$. If $B_{2} \neq A$, then we repeat arguments above. This process can not be infinite because $A$ is a Noetherian $R$-module, so there exist $a_{1}, \ldots, a_{n} \in D$ such that $\left\langle M \cup\left\{a_{1}, \ldots, a_{n}\right\}\right\rangle_{R}=A$ and $A=\langle M\rangle_{R}$

Let $M=\left\{a_{1}, \ldots, a_{k}\right\} \subseteq A$, such that $k \in \mathbb{N}$ is minimal with the properties $A=$ $\langle M\rangle_{R}$ and $k=d_{R}(A)$. Clearly, $A / D=\left\langle a_{1}+D, \ldots, a_{k}+D\right\rangle_{R}$, where $D=\operatorname{Fratt}_{F G}(A)$. It follows that $d_{R}(A / D) \leq d_{R}(A)$. On the other hand, let $\left\{x_{1}, \ldots, x_{m}\right\}$ be a finite subset of $A$ such that $A / D=\left\langle x_{1}+D, \ldots, x_{m}+D\right\rangle_{R}$. Thus $A=\left\langle x_{1}, \ldots, x_{m}\right\rangle_{R}$. Consequently, $m \geq d_{R}(A)$ and $d_{R}(A / D) \geq d_{R}(A)$, so that $d_{R}(A / D)=d_{R}(A)$.

Lemma 2.11. Let $F G$ be the group algebra of a finite group $G$ over a field $F$. Let $A$ be a f.g. FG-module. If $d_{F G}(A)=k$, then $A / F \operatorname{ratt}_{F G}(A)$ is semisimple of finite width. Moreover, each homogeneous component has width at most $k$ and there exists a homogeneous component, whose width is exactly $k$.

Proof. Let $M=\left\{a_{1}, \ldots, a_{k}\right\} \subseteq A$ such that $k \in \mathbb{N}$ is minimal with the property $A=\langle M\rangle_{F G}$. Clearly, each $\left\langle a_{j}\right\rangle_{F G} \cong F G / A n n_{F G}^{\text {left }}\left(a_{j}\right), \operatorname{dim}_{F}\left((F G) a_{j}\right) \leq|G|$ and

$$
\operatorname{dim}_{F}(A)=\operatorname{dim}_{F}\left(F G a_{1}+\cdots+F G a_{k}\right) \leq k|G| .
$$

Since $\operatorname{dim}_{F}(A)$ is finite, $A$ is a Noetherian $F G$-module. Let $\left\{V_{1}, \ldots, V_{s}\right\}$ be a collection of maximal $F G$-submodules of $A$. Let $V=V_{1} \cap \cdots \cap V_{s}$. Using the Remak's theorem we obtain an embedding of $A / V$ into a direct sum $A / V_{1} \oplus \cdots \oplus A / V_{s}$, in which each factor-module $A / V_{j}$ is simple. Since each submodule of a semisimple module is itself semisimple (see [19, Corollary 4.4]), $A / V$ is a semisimple $F G$-module. It follows that $\operatorname{Fratt}_{F G}(A) \leq V$.

Let $0 \neq b \in V \backslash \operatorname{Fratt}_{F G}(A)$. Obviously, $A$ contains a maximal $F G$-submodule $W$ such that $b \notin W$. Put $U=V \cap W$, then $\mathrm{A} / \mathrm{U}$ is embedded into $A / V \oplus A / W$. Using the above arguments, we obtain that $A / U$ is a semisimple $F G$-module, so that again $\operatorname{Fratt}_{F G}(A) \leq U$. In addition, the choice of $U$ implies that $U \neq V$, therefore $\operatorname{dim}_{F}(U)<\operatorname{dim}_{F}(V)$. If $\operatorname{Fratt}_{F G}(A) \neq U$, then we repeat the above argument. This process should be finite, because $\operatorname{dim}_{F}(A)$ is finite. Consequently, $A / \operatorname{Fratt}_{F G}(A)$ is semisimple of finite width.

Finally $d_{R}\left(A / \operatorname{Fratt}_{F G}(A)\right)=d_{R}(A)=k$ by Lemma 2.10 and use Corollary 2.7 .

Consider now the case when a Sylow $p$-subgroup of $G$ is normal.

Lemma 2.12. Let $F G$ be the group algebra of a finite p-group $G$ over a field $F$ of prime characteristic $p$. The length of a $G$-central series of an FG-module $A$ is finite and bounded by $|G|$.

Proof. A semidirect product $A \rtimes G$ is a nilpotent group (see [1, Lemma 3.8, p. 228]). In particular, $A \rtimes\langle g\rangle$ is also nilpotent for each element $g \in G$. 
Set $A_{0}:=A$ and $A_{i}:=(g-1)^{i} A$ for $i \geq 1$. It is easy to see that

$$
\begin{aligned}
& A_{k} \neq\langle 0\rangle, \quad(g-1) A_{k} \neq A_{k}, \quad(0 \leq k \leq|g|-1) \\
& A_{|g|}=(g-1)^{|g|} A=\left(g^{|g|}-1\right) A=\langle 0\rangle .
\end{aligned}
$$

Now we use induction on $|G|$. If $|G|=p$, then $G=\langle g\rangle$ is cyclic and

$$
A=A_{0} \geq A_{1} \geq \cdots \geq A_{|g|}=\langle 0\rangle
$$

is a $G$-central series.

Assume now $|G|>p$. Since a finite $p$-group is nilpotent, $\zeta(G) \neq\langle 1\rangle$ and there exists $1 \neq g \in \zeta(G)$ such that the series (11) is $\langle g\rangle$-central, so that $g \in \mathrm{C}_{G}\left(A_{j} / A_{j+1}\right)$, where $\mathrm{C}_{G}(X)$ is the centralizer of a set $X$ in $G$. An inclusion $g \in \zeta(G)$ shows that each $A_{j}$ is an $F G$-submodule of $A$. Consequently, each factor $A_{j} / A_{j+1}$ can be considered as an $F(G /\langle g\rangle)$-module. Using the inequality $|G /\langle g\rangle|<|G|$ (because $g \neq 1$ ), we can apply the induction hypothesis for $A_{j} / A_{j+1}$. By the induction hypothesis, $A_{j} / A_{j+1}$ has a $(G /\langle g\rangle)$-central series whose length is at most $|G /\langle g\rangle|$. Obviously, factors of such series are also $G$-central for each $0 \leq j \leq k-1$. Thus $A$ has a $G$-central series whose length is at most $|g||G /\langle g\rangle|=|G|$.

\section{Proofs}

Proof of Theorem 1. Let $A$ be an $F G$-module in which $F G$ is the group algebra of a finite group $G$ over the field $F$. Since $A$ has finite $r$-generator property, $A$ has finite $F$-dimension by Lemma 2.8 , so $A$ has a finite series of $F G$-submodules, whose factors are semisimple. Let $B \leq_{F G} A$ such that $d_{F G}(B)=r$ and $D=\operatorname{Fratt}_{F G}(B)$. Corollary 2.10 implies that $d_{F G}(B)=d_{F G}(B / D)=r$ and the factor-module $B / D$ is semisimple and it has finite width by Lemma 2.11. Moreover, every homogeneous component has width at most $r$ and there exists a homogeneous component, whose width is exactly $r$.

Let $V / W$ be a semisimple homogeneous $F G$-factor and set $U:=\operatorname{Fratt}_{F G}(V)$. Obviously, $U \leq W$ and the inequality $d_{F G}(V) \leq r$ implies that $d_{F G}(V / U) \leq r$ by Corollary 2.10. Consequently, $V / U$ is semisimple by Lemma 2.11 and each of its homogeneous components has width at most $r$. The isomorphism

$$
V / W \cong_{F G}(V / U) /(W / U)
$$

implies that $V / W$ has width at most $r$.

Conversely, let $C / E$ be a semisimple homogeneous $F G$-factor, having width $r$. Thus $\operatorname{Fratt}_{F G}(C) \leq E$, so $C / \operatorname{Fratt}_{F G}(C)$ has a homogeneous factor whose width is exactly $r$. By the statements of our theorem, each another homogeneous factor of $C / \operatorname{Fratt}_{F G}(C)$ has width at most $r$. Then $d_{F G}\left(C / \operatorname{Fratt}_{F G}(C)\right)=r$ by Lemma 2.11, and $d_{F G}(C)=r$ by Corollary 2.10, Finally, let $S$ be an $F G$-submodule of $A$. The factor $S / F_{F a t t}(S)$ is semisimple by Lemma 2.11 and each homogeneous factor of $S / \operatorname{Fratt}_{F G}(S)$ has width at most $r$ by parts (i)-(ii) of our theorem. Finally, using 
Lemma 2.11, we obtain that $d_{F G}\left(S / \operatorname{Fratt}_{F G}(S)\right) \leq r$ and $d_{F G}(S) \leq r$, so $A$ has $r$-generator property.

Proof of Theorem 2. Let $F$ be a field of prime characteristic $p$ and let $G$ be a finite group, such that $p$ does not divide $|G|$. If $A$ is an $F G$-module, then $A$ is semisimple (see [19, Corollary 5.15]). It follows that the group ring $F G$ is semisimple. Denote by $n n s_{F}(G)$ the number of pairwise non-isomorphic simple $F G$-modules. This number is finite (see [2, 3]) and can be calculated in the following way.

Let $\xi$ be a primitive $n$-th root of unity, where $n$ is the greatest divisor of $\exp (G)$ which is not divisible by $\operatorname{char}(F)$. Two elements $a, b \in G$ are called $F$-conjugate if there exist $x \in G$ and $m \in \mathbb{N}$ such that $x^{-1} a x=b^{m}$ and the map $\xi \mapsto \xi^{m}$ is extensible to an automorphism of the field $F(\xi)$ fixing the subfield $F$ elementwise. Berman [3] and Witt [24] proved that $\operatorname{nns}_{F}(G)$ is equal to the number of $F$-conjugate classes of $F$-regular elements of $G$ (see [9, Theorem 42.8 p. 306]). If $F$ is algebraically closed, then Brauer [6, 9] shows that $\operatorname{nns}_{F}(G)$ is equal to the number of the conjugate classes of elements of $G$ (see [9, Theorem 40.1 p. 283]). In both cases $n_{n} \sin _{F}(G)$ is bounded by the number of conjugacy classes of $G$.

Since $\operatorname{dim}_{F}(A)$ is finite, $A=\bigoplus_{j=1}^{n} H_{j}$ in which each $H_{j}$ is a homogeneous component of $A$. As we noted above $n \leq n n s_{F}(G)$. The fact that $H_{j}$ is a direct sum of at most $r$ simple submodules follows from Corollary 2.7(i-iii).

Proof of Theorem 3. Clearly, $\operatorname{dim}_{F}(A)$ is finite by Lemma 2.8 and $A$ has a series of $F G$-submodules (see Lemma 2.12)

$$
\langle 0\rangle=Z_{0} \leq Z_{1} \leq \cdots \leq Z_{m-1} \leq Z_{m}=A
$$

whose factors are $p$-central, that is $P \leq \mathrm{C}_{G}\left(Z_{j+1} / Z_{j}\right)$ for each $0 \leq j \leq m-1$. It follows that each $G / \mathrm{C}_{G}\left(Z_{j+1} / Z_{j}\right)$ is a $p$-group, so we can apply Theorem 2 to each of these factors.

\section{REFERENCES}

[1] G. Baumslag. Wreath products and p-groups. Proc. Cambridge Philos. Soc., 55(3):224-231, 1959.

[2] S. D. Berman. On the theory of representations of finite groups. Doklady Akad. Nauk SSSR (N.S.), 86:885-888, 1952 .

[3] S. D. Berman. The number of irreducible representations of a finite group over an arbitrary field. Dokl. Akad. Nauk SSSR (N.S.), 106:767-769, 1956.

[4] A. A. Bovdi. Group rings. (Russian). Kiev.UMK VO, page 155, 1988.

[5] V. A. Bovdi and L. A. Kurdachenko. Some ranks of modules over group rings. Comm. Algebra, 49(3):1225-1234, 2021.

[6] R. Brauer. Zur Darstellungstheorie der Gruppen endlicher Ordnung. Math. Z., 63:406-444, 1956.

[7] I. S. Cohen. Commutative rings with restricted minimum condition. Duke Math. J., 17:27-42, 1950 .

[8] P. M. Cohn. Algebra. Vol. 3. pages xii+474, 1991. 
[9] C. W. Curtis and I. Reiner. Methods of representation theory. Vol. I. Wiley Classics Library. John Wiley \& Sons, Inc., New York, 1990. With applications to finite groups and orders, Reprint of the 1981 original, A Wiley-Interscience Publication.

[10] F. de Giovanni. Infinite groups with rank restrictions on subgroups. Zap. Nauchn. Sem. S.Peterburg. Otdel. Mat. Inst. Steklov. (POMI), 414(Voprosy Teorii Predstavleniı̌ Algebr i Grupp. 25):31-39, 2013.

[11] M. R. Dixon, L. A. Kurdachenko, and I. Y. Subbotin. Ranks of groups. John Wiley \& Sons, Inc., Hoboken, NJ, 2017. The tools, characteristics, and restrictions.

[12] D. Eisenbud. Commutative algebra, volume 150 of Graduate Texts in Mathematics. SpringerVerlag, New York, 1995. With a view toward algebraic geometry.

[13] L. Fuchs and L. Salce. Modules over non-Noetherian domains, volume 84 of Mathematical Surveys and Monographs. American Mathematical Society, Providence, RI, 2001.

[14] R. Gilmer. Two constructions of Prüfer domains. J. Reine Angew. Math., 239/240:153-162, 1969.

[15] R. Gilmer. On commutative rings of finite rank. Duke Math. J., 39:381-383, 1972.

[16] R. Gilmer. The $n$-generator property for commutative rings. Proc. Amer. Math. Soc., 38:477482, 1973.

[17] B. Hartley. A class of modules over a locally finite group. III. Bull. Austral. Math. Soc., 14(1):95$110,1976$.

[18] R. C. Heitmann. Generating ideals in Prüfer domains. Pacific J. Math., 62(1):117-126, 1976.

[19] L. A. Kurdachenko, J. Otal, and I. Y. Subbotin. Artinian modules over group rings. Frontiers in Mathematics. Birkhäuser Verlag, Basel, 2007.

[20] A. I. Mal'cev. On groups of finite rank. Mat. Sbornik N.S., 22(64):351-352, 1948.

[21] A. Matson. Rings of finite rank and finitely generated ideals. J. Commut. Algebra, 1(3):537-546, 2009.

[22] K. Pettersson. Strong $n$-generators and the rank of some Noetherian one-dimensional integral domains. Math. Scand., 85(2):184-194, 1999.

[23] R. G. Swan. n-generator ideals in Prüfer domains. Pacific J. Math., 111(2):433-446, 1984.

[24] E. Witt. Die algebraische Struktur des Gruppenringes einer endlichen Gruppe über einem Zahlkörper. J. Reine Angew. Math., 190:231-245, 1952.

UAEU, Al-Ain, United Arab Emirates

Email address: vbovdi@gmail.com

Department of Algebra and Geometry, School of Mathematics and Mechanics, National University of Dnipro, Ukraine

Email address: 1kurdachenko@i.ua 